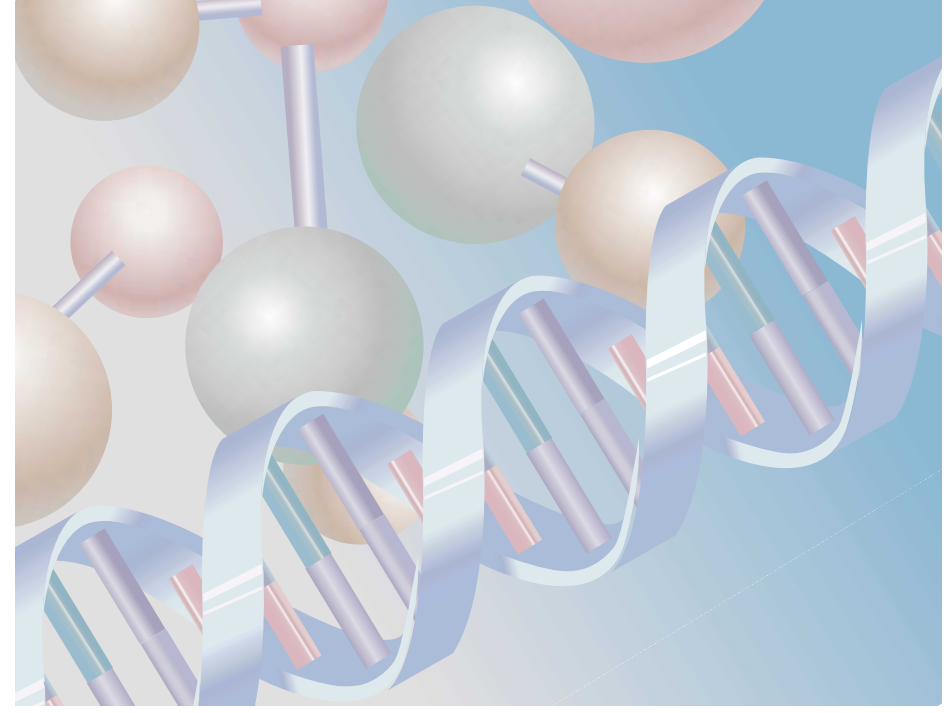

GENE THERAPY

\section{Genetically correct?}

Using an improved gene-transfer protocol, Aiuti and colleagues report in Science the correction of adenosine deaminase (ADA)-deficient severe combined immunodeficiency (SCID) by gene therapy in combination with non-myeloablative conditioning.

ADA-SCID patients have a purine metabolic defect that results in impaired lymphocyte development and function, as well as additional non-immunological abnormalities. Previous gene-therapy trials for this disease have shown that peripheralblood lymphocytes (PBLs) can be corrected genetically by transducing these cells with a retrovirus that encodes the $A D A$ gene, which results in the long-term survival of functional T cells. However, in these trials, patients were also given polyethyleneglycol-conjugated ADA (PEG-ADA) enzyme, so that it was difficult to assess the contribution of the gene therapy to immune reconstitution.

In this study, $\mathrm{CD} 34^{+}$haematopoietic stem cells (HSCs) from two patients for whom PEG-ADA was unavailable were transduced with $A D A$ and infused back into the patients. The patients also received non-myeloablative conditioning a conditioning regimen to achieve immunosuppression and prevent graft rejection without the complete ablation of host haematopoiesis - to provide the transduced cells with a developmental advantage. After transient myelosuppression, haematopoiesis was recovered in these patients the neutrophil count returned to normal, and the number of PBLs, T cells, $\mathrm{B}$ cells and natural killer cells was increased. T-cell development and thymic activity was restored, the T-cell-receptor repertoire was normal, and $\mathrm{T}$ cells had normal proliferative and cytotoxic responses. B-cell functions also seemed to recover. In addition, gene therapy completely restored intracellular ADA activity in PBLs and bone-marrow cells. The two patients are clinically well and remain off enzyme therapy.

The authors carried out real-time PCR to assess the proportion of vector-containing cells in the peripheral blood and bone marrow of the patients. Both sites contained genetically corrected cells of many lineages, including granulocytic, erythroid, megakaryocytic and lymphoid subsets. It was also shown formally that the genetically corrected HSCs retained their repopulation and differentiation capacities.

Therefore, this study establishes that HSC gene therapy combined with non-myeloablative conditioning is effective at restoring lymphoid development and functions, and at correcting the metabolic defect of ADA-SCID, which results in complete reversal of the clinical phenotype in the absence of enzyme-replacement therapy.

Jenny Buckland

6) References and links ORIGINAL RESEARCH PAPER Aiuti, A. et al. therapy combined with nonmyeloablative conditioning. Science 296, 2410-2413 (2002) FURTHER READING Aiuti, A. et al. Immune reconstitution in ADA-SCID after PBL gene therapy and discontinuation of enzyme replacement. Nature Med. 8, 423-425 (2002) | Bordignon, C. and bone marrow for ADA- immunodeficient patients. Science 270, 470-475 (1995) Correction of ADA-SCID by stem-cell gene et al. Gene therapy in peripheral-blood lymphocytes

\section{IN BRIEF}

\section{TUMOUR IMMUNOLOGY}

Tumor-associated B7-H1 promotes T-cell apoptosis: a potential mechanism of immune evasion.

Dong, H. et al. Nature Med. 24 June 2002 (DOI 10.1038/nm730)

Like viruses, tumour cells use many mechanisms to evade host immune responses. Here, Dong et al. show that most human cancers express the co-stimulatory molecule $\mathrm{B} 7-\mathrm{H} 1$, whereas normal tissues do not, with the exception of macrophages. When activated $\mathrm{T}$ cells interact with tumour-associated B7-H1, the T cells die by apoptosis, which seems to be mediated by both interleukin-10 and Fas ligand. This indicates that another method that is used by tumour cells to avoid recognition and destruction by the immune system involves the deletion of activated $\mathrm{T}$ cells at the tumour site. This work has implications for the use of adoptive T-cell therapy to treat cancer. It might be possible to enhance T-cell-mediated killing of B7-H1-positive tumour cells by blockade of $\mathrm{B} 7-\mathrm{H} 1$ using monoclonal antibodies or soluble inhibitors.

\section{INNATE IMMUNITY}

Activation of Drosophila Toll during fungal infection by a blood serine protease.

Ligoxygakis, P., Pelte, N., Hoffmann, J. A. \& Reichart, J.-M. Science 297, 114-116 (2002)

In Drosophila, a cleaved form of Spaetzle is the extracellular ligand for the Toll pathway in both embryonic development and immune responses to fungal pathogens. However, the upstream events that link infection to the cleavage of Spaetzle remain undefined. Here, Ligoxygakis et al. show - by screening for mutants that suppress the constitutive activation of the anti-fungal pathway in Necrotic mutant flies - that persephone ( $p s h)$, a serine protease, is a central component of the fungalmediated activation of Toll. The upstream pattern-recognition molecule that activates the protease function of $p s h$ remains to be identified.

\section{HIV}

\section{Epistatic interaction between KIRDS1 and $H L A-B$} delays the progression to AIDS.

Martin, M. P. et al. Nature Genet. 22 July 2002 (DOI 10.1038/ng934)

Natural killer (NK)-cell responses are regulated by the balance of signals that are received from activating and inhibitory receptors. Killer immunoglobulin-like receptors (KIRs) are expressed on human NK cells and interact with HLA class I molecules on target cells. In this study, Martin et al. report that the activating KIR allele KIRDS1, in association with HLA-B Bw4-80Ile - an $H L A-B$ allele that has an isoleucine residue at position $80-$ is associated with a delay in the development of AIDS in HIV-1positive patients. The absence of HLA-B Bw4-80Ile is associated with a more rapid progression to AIDS, whereas the absence of KIRDS1 has no effect. The identification of this interaction might provide a new approach for the development of vaccines or therapeutic agents. 